\title{
Differential Evolution Algorithm for Optimizing Virtual Machine Placement Problem in Cloud Computing
}

\author{
Amol C. Adamuthe \\ Department of Information Technology, Rajarambapu Institute of Technology, Rajaramnagar, MS, India \\ E-mail: amol.admuthe@gmail.com \\ Jayshree T. Patil \\ Department of Computer Engineering, Rajarambapu Institute of Technology, Rajaramnagar, MS, India \\ E-mail: jtpati11010@gmail.com
}

Received: 03 July 2017; Accepted: 11 September 2017; Published: 08 July 2018

\begin{abstract}
Primary concern of any cloud provider is to improve resource utilization and minimize cost of service. Different mapping relations among virtual machines and physical machines effect on resource utilization, load balancing and cost for cloud data center. Paper addresses the virtual machine placement as optimization problem with resource constraints on CPU, memory and bandwidth. In experimentations, datasets are formed using random data generator. Paper presents random fit algorithm, best fit algorithm based on resource wastage and an evolutionary algorithm- Differential Evolution. Paper presents results of Differential Evolution algorithm with three different mutation approaches. Results show that Differential Evolution algorithm with DE/best/2 mutation operator works efficient than basic DE, best fit and random fit algorithms.
\end{abstract}

Index Terms-Differential Evolution Algorithm (DE), Virtual machine placement problem (VMP), Best fit, Random fit.

\section{INTRODUCTION}

Cloud computing is a technology that provides ondemand services over the internet such as computing resources, data or software. The cloud computing has four deployment models i.e. public, private, hybrid and community. Software as a Service (SaaS), Platform as a Service (PaaS) and Infrastructure as a Service (IaaS) are the three important service models in cloud computing. Cloud providers have large computing resources in large data centers which are available to users on a per-use basis [1]. A data center is a group of physical machines or hosts. Each physical machine has computing capacity, memory, bandwidth, and storage capacity [2].

Today, cloud computing is one of the most explosively expanding technologies in the computing industry. Therefore, the number and the scale of cloud service providers greatly increased. More data centers mean more energy supply and higher operating costs. It places a heavy burden on both environment and energy resources. Optimization is essential for cloud computing providers to provide a good value to potential customers. The placement of virtual machine in cloud infrastructure is one of the main research problems. The execution or placement of virtual machine on each physical machine of the data center is the process which is called virtual machine placement. In other words, placement of virtual machine is to choice the most appropriate host designed for the virtual machine process. The placement of virtual machine has many objectives like a minimum number of physical machines used, effective usage of power consumption and resource utilization such as CPU, memory, and bandwidth. The problem is how to accomplish utmost of these properties in a allocating of the virtual machine structure, to have extra efficient, little overhead, short cost and scalable allocates of virtual machine in the cloud data centers. However, approximately of these objectives are in conflicting with each other and wholly of them may not completely be attained in one allocating scheme [2-4].

In this paper virtual machine and physical machine is measured in three-dimensional item such as CPU size, memory size and storage size. The allocation of the virtual machines taking place physical machine is similar to the $3 \mathrm{D}$ bin packing problem. In three-dimensional bin packing problem, a group of 3D items is necessary to be placed inner side 3D bins. The main goal of this problem is to pack as more as possible items in the bins, so that the minimum count of bins are essential. In bin packing problem two items can be placed into bins beside each other or one on top of the other but this is impossible operation in a placement of VMs. The placement of virtual machine problem is similar to Vector Packing Problem and it is also an NP-Hard problem [5, 6].

The placement of virtual machine problem is constrained optimization problem, where the objective is to allocate virtual machine to physical machine by satisfying given constraints. The problem is ' $\mathrm{P}$ ' problem 
means when the problem size is small i.e. quantity of physical machines (PMs) and virtual machines (VMs) are less. Problem complexity increases with the size of a problem. When the number of virtual machines and physical machines are big enough, the situation becomes difficult to manage. Even in the automatic calculation of the placement plan, the combinations are large for a given number of virtual machines and physical machines. Such an enormous solution space creates it an NP problem. Literature reports that it is nearly unbearable for brute force algorithms to complete the physical and virtual machine mapping optimally within acceptable time. Therefore, the needs for intelligent placement of heuristics to narrow the search for solutions to get close to the best placement plan [7]. Heuristic algorithms such as Genetic Algorithms, Particle swarm optimization, Ant colony optimization are good candidates which has the ability to find sub-optimal solutions in finite time.

In cloud computing work, virtual machine placement problem is studied from different perspectives. The problem is studied with different objectives, constraints and addressed by different traditional and heuristic algorithms [2, 3]. In this paper, the problem is expressed a single-objective optimization problem with resource constraints. Objective of this paper is to apply Differential Evolution algorithm and identify suitable operators for VMP. The results are compared with random fit algorithm and best- fit algorithm.

The paper is arranged as follows: Section II presents the background of virtual machine placement problem and different problems solved using Differential Evolution algorithm. Section III describes the virtual machine placement problem. Section IV describes the best fit, random fit and Differential Evolution algorithm. Section V presents dataset generator for problem instances and results obtained by best fit, random fit and Differential Evolution algorithm. Finally, the conclusions of our study are outlined in Section VI.

\section{RELATED WORK}

In cloud computing work, the virtual machine placement has been broadly studied. Some of the important issues addressed in literature are energy efficiency, specific architectures and performance of placement algorithms comparing with different methods [8]. The problem is formulated with heterogeneous as well as homogeneous virtual machine and physical machine $[3,9]$.

Different researchers considered different objective function for placement of virtual machine problem in cloud computing such as, minimize energy or power consumption, minimize number of physical machines [4$6,9,10-12]$, minimize network traffic $[6,5,10,13,14]$, minimize economical cost or operational cost [5, 15-17], maximize performance and resource utilization, reduce wastage resource $[4,7,18]$, improve utilization of cloud resources, maximizing providers' revenue, decreasing cost for cloud providers and growing the return on investment (ROI), improving load balancing, high performance, throughput and response time [7].

Many researchers found that why energy efficiency is important in cloud data infrastructure [9]. They have firstly identified sources of energy wastage in data centers then to overcome energy wastage they made a plan of work. This plan of work analyses how to manage energy efficiency in data center and design problem formulation for this issue.

In literature, there are many techniques used to solve placement problem of virtual machines such as constraint programming, stochastic integer programming, greedy algorithm, heuristics based algorithms, game theory based algorithms and graph theory based algorithms.

The constraint programming is beneficial for combinatorial search problems and its solutions strictly fulfill the constraints on dealings among variables. Stochastic integer programming is applicable for forming optimization problems. Author proposed optimal virtual machine placement to deliver resources of several cloud providers and minimize cost for placing the virtual machine on a physical machine [3, 9].

Greedy algorithm is one of the traditional algorithms used for solving the placement problem of the virtual machines. These algorithms necessity fewer polynomial time complexity than the meta-heuristics algorithm and it can be executed straightforwardly. In virtual machine placement problem first-fit decreasing is one of the famous greedy algorithms, placement of virtual machine is sequential. Another traditional algorithm is the best fit, random fit and next fit. In random fit algorithm randomly select a physical machine to place virtual machine on it.

Heuristics algorithms are considered as new good candidates who are able to find near optimum solution for the problem [19]. In [20], authors proposed genetic algorithm for single objective virtual machine placement problem. The objective is cost minimization for physical machines. Results show that genetic algorithm is better than the first-fit decreasing algorithm. In many papers it is found that heuristic based techniques are better $[4,5,7$, $12,21-25]$.

Paper [26], tested performance of three evolutionary algorithms namely Differential Evolution (DE), Genetic Algorithms (GA) and Particle Swarm Optimization (PSO). Results show that as compared to GA and PSO, the DE has the least clustering and re-initialization effect. Also DE did not have any influence (that is a dominant influence) on population. Different problems are solved using Differential Evolution algorithm such as scheduling and resource allocation [27-32], clustering [33, 34], scheduling of the hydro power generator [31]. Santucci V. et al. [28], proposed Differential Evolution for variation flow-shop scheduling problem with the overall flow time criterion. For the mutation operator, they used the biased selection strategy, mimetic restart procedure, and heuristic based initialization. To do the analysis they have done the performance comparisons with the widely accepted benchmark suite. Zhao M. et al. [35], has done the research on the constrained optimization problem using multi-objective Differential Evolution algorithm. 


\section{VIRTUAL Machine Placement PRoblem}

In cloud computing, virtual machine placement is one of the most demanding problems. It has a direct effect on the performance, resource utilization and power consumption of the data centers and can reduce the maintenance cost of the data centers for cloud providers. Numerous VM placement schemes are designed and proposed for VM placement in the cloud computing environment aimed to improve various factors affecting the data centers, the VMs and their executions.

The objective of this paper is to minimize the required number of physical machines to place all the virtual machines by satisfying resource constraints. In literature, many researchers worked to achieve similar objective [4$6,9,12-14]$. The minimization of required physical machines leads to maximize CPU, memory, bandwidth utilization, electricity and cost minimization.

In this problem formulation, we have considered physical and a virtual machine with three attributes namely CPU i.e. computing capacity, memory and bandwidth. The set of a physical machines and virtual machines with their resource capacity are known.

The objective of placement virtual machine problem is to minimize set of physical machines required to place all virtual machines by following constraints,

\section{Capacity Constraints:}

For each dimension of a physical machine, the summation of the resource requirements of all virtual machines allocated should be fewer than or equivalent to the total accessible capacity of that physical machine. VM $i$ place on PM $m$ indicates 1 otherwise 0 .

\section{Placement Guarantee Constraints:}

All the virtual machines must be located on the physical machine.

Capacity constraint and placement guarantee constraints are hard constraints which must be fulfilled. Violation of these constraints (also called as conflicts) will reason the solution to be infeasible.

\section{ALOGRITHMS}

This section presents algorithms experimented to solve virtual machine placement problem. This paper presents comparison of best fit algorithm, random fit algorithm and Differential Evolution algorithm.

\section{A. Random Fit Algorithm}

In the random fit algorithm, allocates random physical machine which is big enough for allocating virtual machine with satisfying all constraints [36]. Fig. 1 shows random fit algorithm for solving virtual machine placement problem.

\section{B. Best Fit Algorithm}

In best-fit algorithm, allocates the available smallest physical machine which is big enough for allocating virtual machine with satisfying all constraints. The smallest physical machine is which results in minimum resource wastage [36].

The meaning of resource wastage and its calculation technique are taken from [37]. The remaining resources accessible on each host may differ greatly depending on the virtual machine placement solution. Unbalanced remaining resources may stop any further VM placement, therefore wasting computing resources. The cross-shaded area in the figure represents the remaining resources that can be used for upcoming allocation.

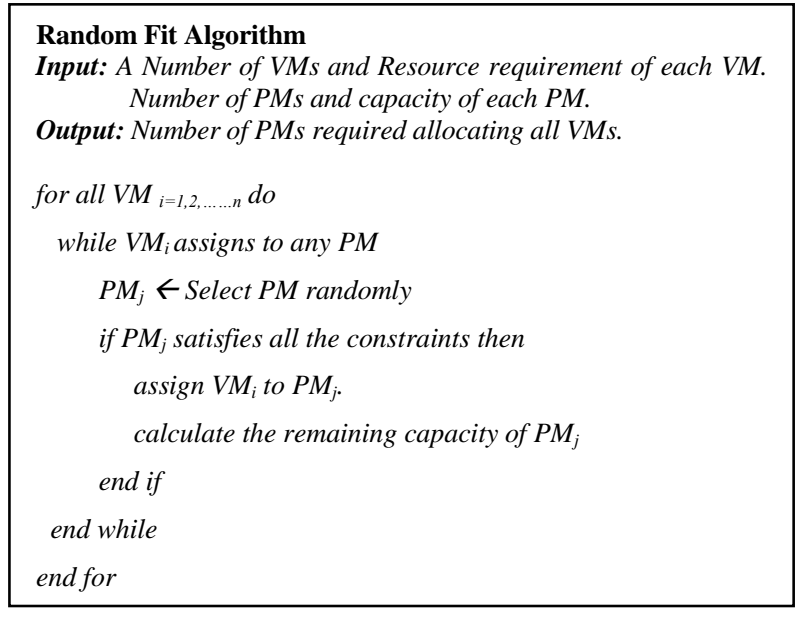

Fig.1. Random fit algorithm

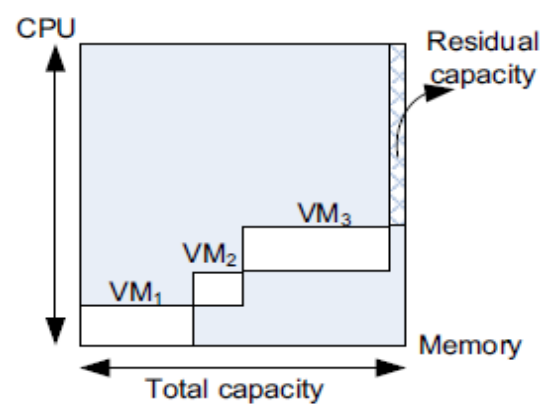

Fig.2. Resource wastage [37]

In the example of Fig. 2, the host has many idle CPU capacities, but the available memory is very small, causing the host to accept any new VMs due to insufficient memory. To balance the usage of resources in different dimensions, use the following symbols to calculate the potential cost of wasting resources. $R_{i}$ denotes the normalized remaining resource with dimension $i$. The subscript $k$ is used to recognize the dimension with the least normalized remaining capacity and the remaining resources wasted on the server are calculated as the sum of the minimum normalized remaining resources and the difference between the other resources [37]. 
The relationship among $\mathrm{W}$ and resource remaining is linear. Extra remaining resource variances in dissimilar dimensions, the more resources are unused.

$$
W=\sum_{i \neq k}\left(R_{i}-R_{k}\right)
$$

Fig. 3 presents best fit algorithm for solving virtual machine placement problem.

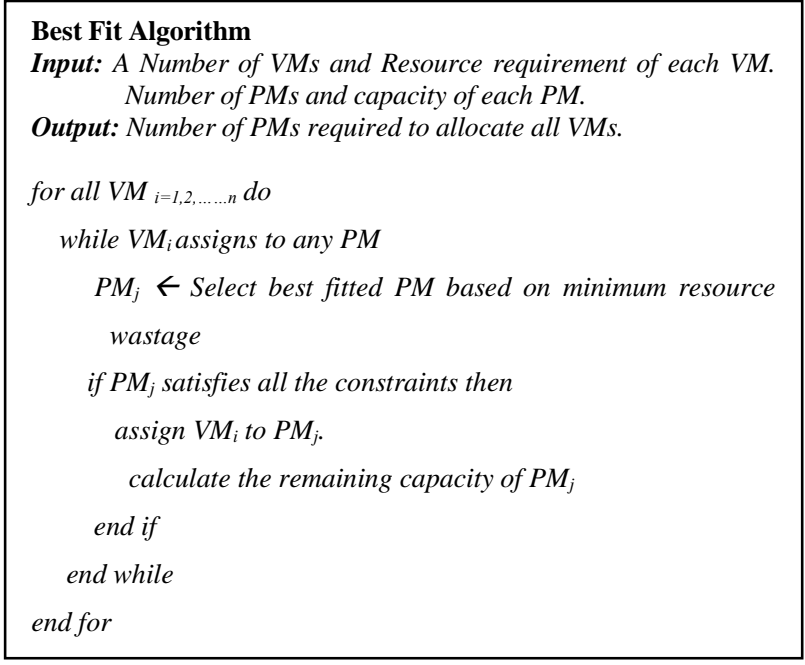

Fig.3. Best fit algorithm

\section{Differential Evolution Algorithm}

Differential Evolution algorithm is an evolutionary algorithm. The DE algorithm is used for global optimization. The main advantage of DE algorithm is improvement takes place in the solution after individually iteration. Differential Evolution's theoretical framework is simple and it has or requires the fewer algorithm parameters but that performs well in convergence. In the Differential Evolution solution space is denoted in DDimensional vectors and this space is denoted as a real number. Differential Evolution algorithm fulfills four requirements of user for minimization techniques such as ability to handle nonlinear, non-differentiable, multimodal cost functions, parallelizability to cope with computation intensive cost functions, ease of usage and good convergence properties [38]. Fig. 4 presents DE algorithm.

As compared to the Differential Evolution (DE) algorithm, genetic algorithm (GA), particle swarm optimization (PSO) has two main advantages such as improvement takes place in the solution immediately after each iteration and requires fewer number of algorithm specific parameters [26].

In the DE Initial vector is the size of $n$ in Ddimensional. The new solution is generated by merging the several candidate solutions. New population generation is done in repeated cycle by using the three DE operators such as mutation, crossover, and selection. Generating the trial vector is a key process in DE, the generation of the trial vector by using the candidate vectors is done by using 2 steps:
- By using the three randomly selected candidate vectors, a Mutant vector is generated using the mutation operator.

- By doing the crossover of the mutant vector and candidate vector the trial vector generated

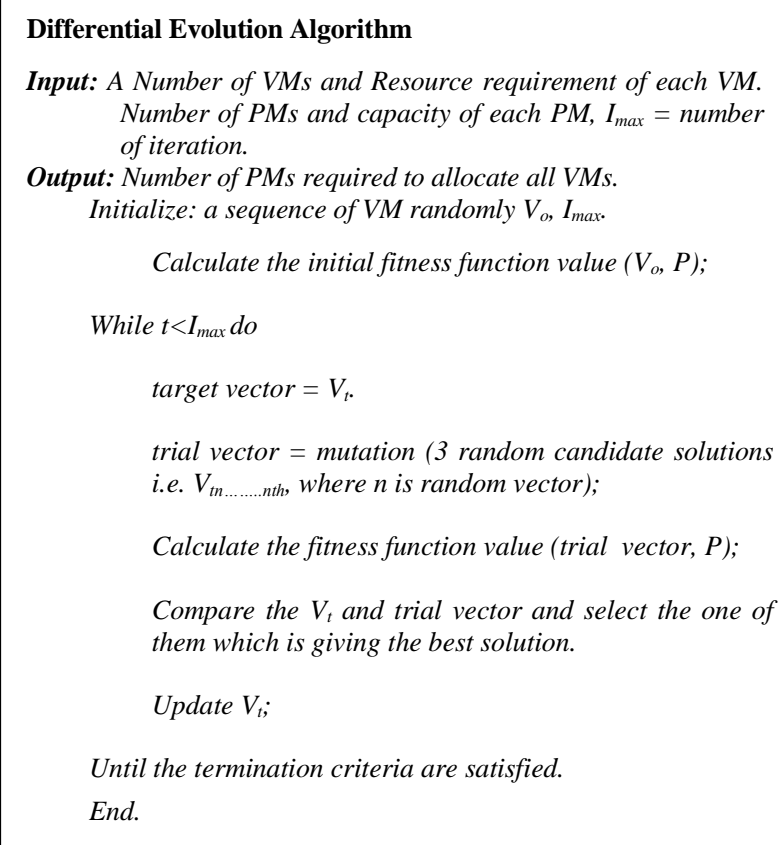

Fig.4. Differential Evolution algorithm

In $\mathrm{DE}$ algorithm, different variations are classified into ' $\mathrm{DE} / \mathrm{x} / \mathrm{y} / \mathrm{z}$ '. In this notation $\mathrm{x}$ represents vector to be mutated can be randomly or best, $y$ represents numbers of variance vectors are used and $\mathrm{z}$ represents crossover scheme, present different is 'bin'. Using this notation in DE algorithm use basic DE strategy is used i.e. 'DE/rand/1/bin'.

$\mathrm{DE} / \mathrm{rand} / 1$ : In this, indices are selected randomly, so mutation operator is totally based on the random nature of the indices.

$$
X_{n e w, g}=X_{r 1, g}+F \cdot\left(X_{r 2, g}-X_{r 3, g}\right)
$$

Where $X_{\text {new, }}$ is new vector generated by the mutation operator. $\mathrm{r} 1, \mathrm{r} 2, \mathrm{r} 3$ is randomly generated indices and $\mathrm{F}$ is fiddle factor.

\section{EXPERIMENTAL DETAILS, RESUlTS AND DISCUSSION}

In this section, provides detail specifications of problem instances and the results found by best fit, random fit and Differential Evolution algorithm.

\section{Data Generator:}

Eight problem instances are randomly generated to compare the performance of algorithms. Random data generator program is written in $\mathrm{C}$ programming language.

Characteristics of instances 
1. For experimentation we assumed heterogeneous physical machines and virtual machines.

2. The PM and VM attribute requirements were created as a maximum or minimum combination of different ranges uniformly distributed random numbers.

3. Datasets contains combination of CPU intensive, memory intensive and bandwidth intensive virtual machines. The VM requirements were generated as a mix of different ranges of uniformly distributed random numbers as follows:

$$
\begin{aligned}
& <0.7-0.9,0.1-0.3,0.1-0.3>: 30-35 \% \mathrm{VMs} \\
& <0.1-0.3,0.7-0.9,0.1-0.3>: 30-30 \% \mathrm{VMs} \\
& <0.1-0.3,0.1-0.3,0.7-0.9>: 30-35 \% \mathrm{VMs}
\end{aligned}
$$

4. Performance of evolutionary algorithms may vary with the problem size. Performance of Differential Evolution algorithm is tested on small instance with 20 virtual machines and large instance with 250 virtual machines.

Price and Storn proposed DE/rand/1 mutation operator in DE framework. This operator is most widely used by many researchers. In this paper, performance of basic mutation operator is compared with two different mutation operators described in [39].

1) DE/best/2: In this, indices are not selected randomly, the best index is selected from the population which is best individuals. So, mutation operator is not based on the random nature of the indices.

$$
X_{n e w, g}=X_{b e s t}+F \cdot\left(X_{r 1, g}-X_{r 2, g}\right)+F \cdot\left(X_{r 3, g}-X_{r 4, g}\right)
$$

Where $X_{b e s t, g}=$ is indicates the best individuals.

2) DE/current-to-best/1: In this, indices are not selected randomly, the best individuals are selected from the current population. So, the mutation operator is based on the current population to best individual's nature of the indices.

$$
X_{\text {new }, g}=X_{\text {current }, g}+F \cdot\left(X_{\text {best }}-X_{\text {current }, g}\right)+F \cdot\left(X_{r 2, g}-X_{r 3, g}\right)
$$

Differential Evolution algorithm with basic mutation operator, DE/rand/1, provides good solutions to VMP problem. Table 1 shows comparison of three different mutation operators of Differential Evolution algorithm for the virtual machine placement problem described in section III. The best results obtained in 10 runs are reported with respect to iterations. Differential Evolution algorithm with $\mathrm{DE} / \mathrm{Best} / 2$ mutation operator gives better results and shows better convergence than other mutation operators.
Table 1. Convergence of Differential Evolution Algorithm with

\begin{tabular}{|c|c|c|c|c|c|}
\hline $\begin{array}{c}\text { No. } \\
\text { of } \\
\text { VMs }\end{array}$ & Algorithm & $\begin{array}{l}\text { PMs } \\
\text { used }\end{array}$ & $\begin{array}{c}\mathrm{CPU} \\
\text { wastage }\end{array}$ & $\begin{array}{l}\text { Memory } \\
\text { wastage }\end{array}$ & $\begin{array}{c}\text { Bandwidth } \\
\text { wastage }\end{array}$ \\
\hline \multirow{3}{*}{20} & DE/best/2 & 3 & 867 & 47 & 393 \\
\hline & $\begin{array}{c}\text { DE/current } \\
\text {-to-best/1 }\end{array}$ & 4 & 717 & 181 & 649 \\
\hline & DE/rand/1 & 5 & 1155 & 1254 & 390 \\
\hline \multirow{3}{*}{40} & DE/best/2 & 8 & 1021 & 2615 & 420 \\
\hline & $\begin{array}{l}\mathrm{DE} / \text { current } \\
\text {-to-best/1 }\end{array}$ & 8 & 1021 & 2615 & 420 \\
\hline & DE/rand/1 & 8 & 1400 & 3106 & 828 \\
\hline \multirow{3}{*}{60} & DE/best/2 & 11 & 1226 & 3668 & 1359 \\
\hline & $\begin{array}{c}\text { DE/current } \\
\text {-to-best/1 }\end{array}$ & 14 & 1513 & 2774 & 2163 \\
\hline & DE/rand/1 & 16 & 4059 & 2777 & 3201 \\
\hline \multirow{3}{*}{80} & DE/best/2 & 13 & 2685 & 602 & 1408 \\
\hline & $\begin{array}{c}\text { DE/current } \\
\text {-to-best/1 }\end{array}$ & 13 & 3945 & 1763 & 1796 \\
\hline & DE/rand/1 & 14 & 4722 & 2197 & 1876 \\
\hline \multirow{3}{*}{100} & DE/best/2 & 22 & 4350 & 5937 & 3907 \\
\hline & $\begin{array}{c}\text { DE/current } \\
\text {-to-best/1 }\end{array}$ & 23 & 5201 & 5201 & 3638 \\
\hline & DE/rand/1 & 24 & 3950 & 6708 & 3752 \\
\hline \multirow{3}{*}{150} & DE/best/2 & 30 & 5112 & 8937 & 4601 \\
\hline & $\begin{array}{c}\text { DE/current } \\
\text {-to-best/1 }\end{array}$ & 31 & 5475 & 6289 & 4526 \\
\hline & DE/rand/1 & 31 & 5526 & 8066 & 4502 \\
\hline \multirow{3}{*}{200} & DE/best/2 & 40 & 5717 & 8536 & 5378 \\
\hline & $\begin{array}{c}\text { DE/current } \\
\text {-to-best/1 }\end{array}$ & 41 & 7113 & 10070 & 6737 \\
\hline & DE/rand/1 & 42 & 6311 & 10993 & 6910 \\
\hline \multirow{3}{*}{250} & DE/best/2 & 47 & 9864 & 9179 & 5446 \\
\hline & $\begin{array}{c}\text { DE/current } \\
\text {-to-best/1 }\end{array}$ & 49 & 11931 & 10108 & 5806 \\
\hline & $\mathrm{DE} / \mathrm{rand} / 1$ & 51 & 7960 & 10381 & 6737 \\
\hline
\end{tabular}
Different Mutation Operators

\begin{tabular}{|c|c|c|c|}
\hline Iteration & $\mathrm{DE} / \mathrm{rand} / 1$ & $\begin{array}{c}\text { DE/current-to- } \\
\text { best/1 }\end{array}$ & DE/Best/2 \\
\hline 100 & 53 & 51 & $\mathbf{5 0}$ \\
\hline 500 & 52 & 50 & $\mathbf{4 8}$ \\
\hline 1000 & 51 & 49 & $\mathbf{4 7}$ \\
\hline 2000 & 50 & 47 & $\mathbf{4 6}$ \\
\hline 5000 & 49 & 46 & $\mathbf{4 5}$ \\
\hline 10000 & 47 & 45 & $\mathbf{4 4}$ \\
\hline 15000 & 46 & 45 & $\mathbf{4 3}$ \\
\hline 20000 & 46 & 44 & $\mathbf{4 3}$ \\
\hline Figures in bold indicates best values. & & \\
\hline
\end{tabular}

Table 2. Results of Differential Evolution Algorithm with Different Mutation Operators

Differential Evolution algorithm is tested on eight problem instances with virtual machines varying from 20 to 250. Table 2 shows comparison of three different mutation operators of Differential Evolution algorithm, where the best results in 10 runs are reported. The algorithms are executed up to 100 iterations. Table 2 presents number of physical machines required to fit given virtual machines, CPU wastage, memory wastage and bandwidth wastage. DE/best/2 mutation operator gives better results than other two operators for six 
instances with respect to number of physical machines used. Performance of $\mathrm{DE} / \mathrm{best} / 2$ is same or similar to DE/current-to-best/1 mutation operator for problem instances with 40 and $80 \mathrm{VMs}$ respectively. For most of instances, performance of DE/current-to-best/1 is better than $\mathrm{DE} / \mathrm{rand} / 1$ mutation operator.

Table 3 shows comparison of Differential Evolution algorithms with basic operators and best fitted operator with best fit algorithm and random fit algorithm.

Table 3. Comparisons of Differential Evolution, Random fit, and Best fit Algorithm

\begin{tabular}{|c|c|c|c|c|c|}
\hline $\begin{array}{c}\text { No. } \\
\text { of } \\
\text { VMs }\end{array}$ & Algorithm & $\begin{array}{l}\text { PMs } \\
\text { used }\end{array}$ & $\begin{array}{c}\mathrm{CPU} \\
\text { wastage }\end{array}$ & $\begin{array}{l}\text { Memory } \\
\text { wastage }\end{array}$ & $\begin{array}{l}\text { Bandwidth } \\
\text { wastage }\end{array}$ \\
\hline \multirow{4}{*}{20} & DE/best/2 & 3 & 867 & 47 & 383 \\
\hline & DE/rand/1 & 5 & 1155 & 1254 & 390 \\
\hline & Best fit & 7 & 1207 & 2290 & 876 \\
\hline & $\begin{array}{l}\text { Random } \\
\text { fit }\end{array}$ & 10 & 3521 & 4678 & 1398 \\
\hline \multirow{4}{*}{40} & DE/best/2 & 8 & 1021 & 2615 & 420 \\
\hline & DE/rand/1 & 8 & 1400 & 3106 & 828 \\
\hline & Best fit & 12 & 1861 & 3938 & 1452 \\
\hline & $\begin{array}{l}\text { Random } \\
\text { fit }\end{array}$ & 20 & 6897 & 6044 & 4342 \\
\hline \multirow{4}{*}{60} & DE/best $/ 2$ & 11 & 1226 & 3668 & 1359 \\
\hline & DE/rand/1 & 16 & 4059 & 2777 & 3201 \\
\hline & Best fit & 18 & 4193 & 2526 & 3270 \\
\hline & $\begin{array}{c}\text { Random } \\
\text { fit }\end{array}$ & 25 & 9309 & 8367 & 5236 \\
\hline \multirow{4}{*}{80} & DE/best/2 & 13 & 2685 & 602 & 1408 \\
\hline & DE/rand/1 & 14 & 4722 & 2197 & 1876 \\
\hline & Best fit & 21 & 7002 & 4028 & 3093 \\
\hline & $\begin{array}{c}\text { Random } \\
\text { fit }\end{array}$ & 30 & 15230 & 9387 & 5589 \\
\hline \multirow{4}{*}{100} & $\mathrm{DE} / \mathrm{best} / 2$ & 22 & 4350 & 5937 & 3907 \\
\hline & DE/rand/1 & 24 & 3950 & 6708 & 3752 \\
\hline & Best fit & 28 & 4791 & 5911 & 4123 \\
\hline & $\begin{array}{c}\text { Random } \\
\text { fit }\end{array}$ & 40 & 14304 & 15010 & 7861 \\
\hline \multirow{4}{*}{150} & DE/best/2 & 30 & 5112 & 8937 & 4601 \\
\hline & DE/rand/1 & 31 & 5526 & 8066 & 4502 \\
\hline & Best fit & 39 & 6326 & 7668 & 6005 \\
\hline & $\begin{array}{c}\text { Random } \\
\text { fit }\end{array}$ & 60 & 24108 & 23826 & 11608 \\
\hline \multirow{4}{*}{200} & DE/best/2 & 40 & 5717 & 8536 & 5378 \\
\hline & DE/rand/1 & 42 & 6311 & 10993 & 6910 \\
\hline & Best fit & 55 & 8465 & 11880 & 9051 \\
\hline & $\begin{array}{l}\text { Random } \\
\text { fit }\end{array}$ & 80 & 28840 & 32252 & 16749 \\
\hline \multirow{4}{*}{250} & DE/best/2 & 47 & 9864 & 9179 & 5446 \\
\hline & DE/rand/1 & 51 & 7960 & 10381 & 6737 \\
\hline & Best fit & 64 & 10791 & 10618 & 7754 \\
\hline & $\begin{array}{l}\text { Random } \\
\text { fit }\end{array}$ & 100 & 38540 & 40010 & 17665 \\
\hline
\end{tabular}

Result from Table 2, Table 3 and Fig. 5 shows that Differential Evolution algorithm with all three mutation operator gives better results than best fit algorithm and random fit algorithm for all the eight instances of VMP. Fig. 6, Fig. 7 and Fig. 8 shows the comparison of selected algorithms with respect to resource wastage. Differential
Evolution algorithm with DE/best/2 mutation operator minimizes the number of physical machines used and resource wastage viz. CPU, memory and bandwidth.

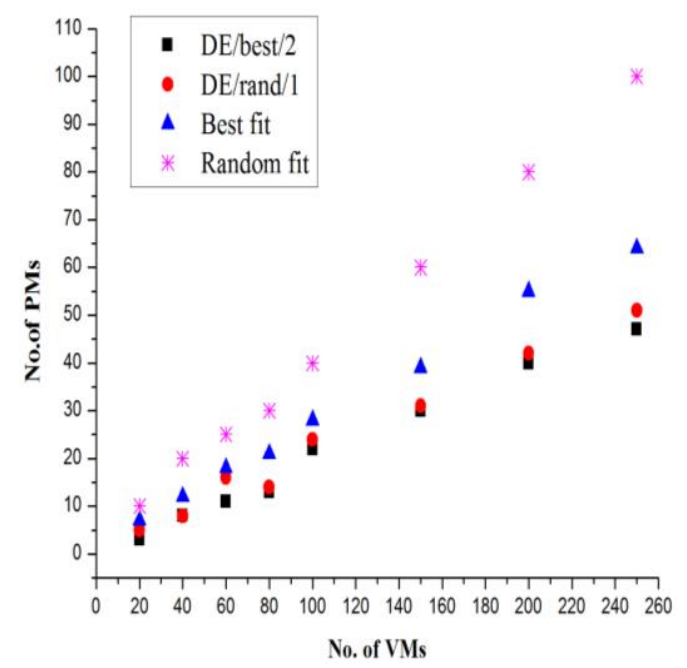

Fig.5. Results of selected algorithms for VMP problem for 8 instances

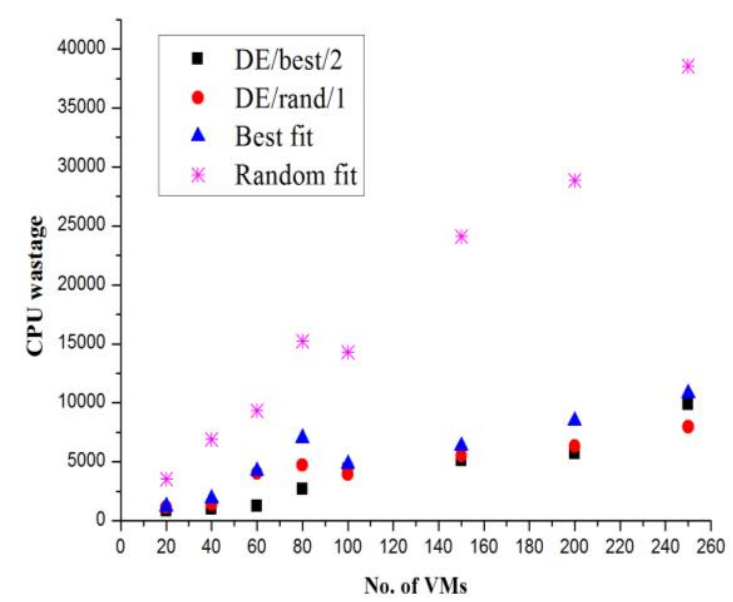

Fig.6. Comparison of selected algorithms on CPU wastage level

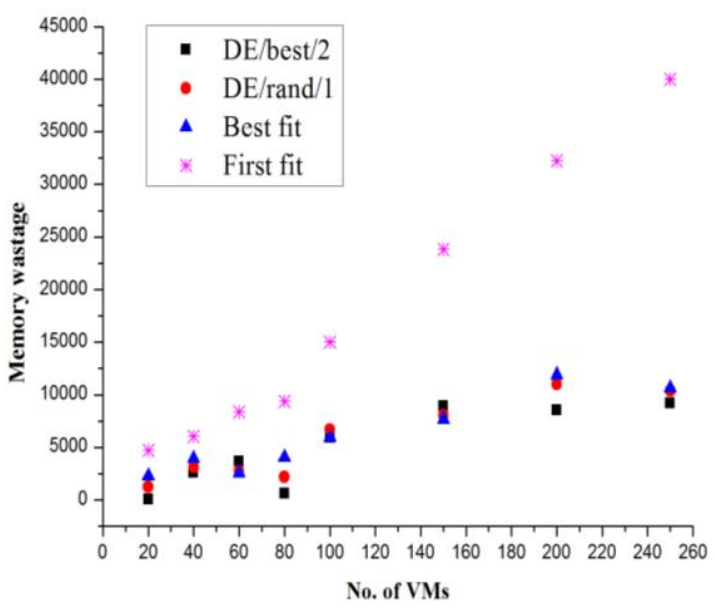

Fig.7. Comparison of selected algorithms on memory wastage level 


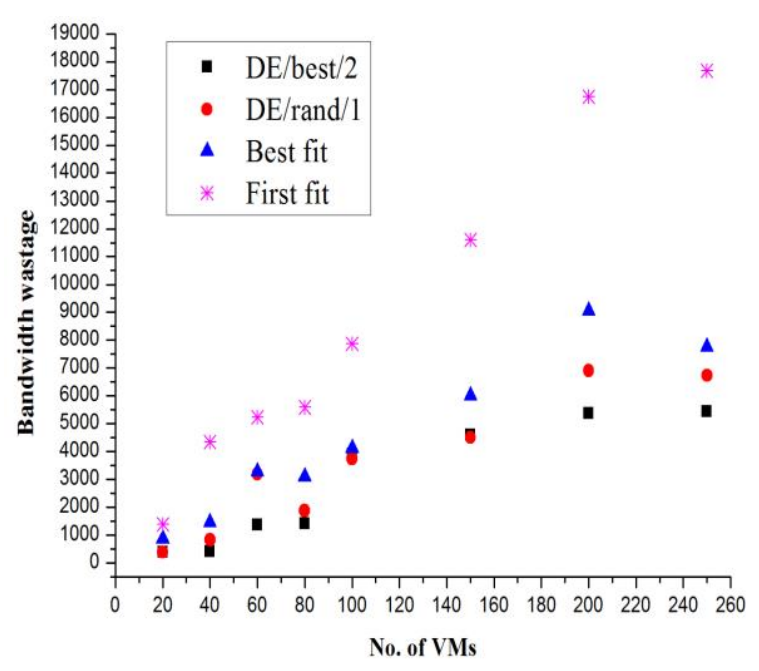

Fig.8. Comparison of selected algorithms on bandwidth wastage level

\section{CONCLUSIONS}

This paper presents virtual machine placement problem as constrained optimization problem. The objective is to minimize required number of physical machines to place all the virtual machines by satisfying resource constraints and with placement guarantee. Paper shows experimentation with Differential Evolution algorithms with three different mutation operators, best fit algorithm and random fit algorithm. Differential Evolution algorithm with selected three mutation operators give better results than best fit algorithm and random fit algorithm for all the eight problem instances. Results show that DE with DE/best/2 mutation operator gives better solutions and faster convergence than DE/currentto-best/1and DE/rand/1.

\section{REFERENCES}

[1] G. Lee, "Resource Allocation and Scheduling in Heterogeneous Cloud Environments", Technical Report No. UCB/EECS-2012, 2012.

[2] A. Kumar, C. Sathasivam, P. Periyasamy, "Virtual machine placement in cloud computing", Indian Journal of Science and Technology, 2016, vol. 9, no. 29.

[3] M. Masdari, SS. Nabavi, V. Ahmadi, "An overview of virtual machine placement schemes in cloud computing," Journal of Network and Computer Applications, 2016, vol. 66, pp. 106-127.

[4] Y. Gao, H. Guan, Z. Qi, Y. Hou, L. Liu., "A multiobjective ant colony system algorithm for virtual machine placement in cloud computing," Journal of Computer and System Sciences, 2013, vol. 79, no .8, pp. 1230-1242.

[5] R. Sookhtsaraei, M. Madani, A. Kavian, "A multi objective virtual machine placement method for reduce operational costs in cloud computing by genetic," International Journal of Computer Networks and Communications Security, 2014, vol. 2, no. 8, pp. 250256.

[6] J. Dong, X. Jin, H. Wang, Y. Li, P. Zhang, S. Cheng, "Energy-saving virtual machine placement in cloud data centers," In Cluster, Cloud and Grid Computing, $13^{\text {th }}$ IEEE/ACM International Symposium, 2013, pp. 618-624.

[7] A. C. Adamuthe, R. M. Pandharpatte, G. T. Thampi,
"Multiobjective virtual machine placement in cloud environment," In Cloud \& Ubiquitous Computing \& Emerging Technologies, IEEE International Conference, 2013, pp. 8-13.

[8] F. Lopez-Pires, B. Baran, "Virtual machine placement literature review", arXiv preprint arXiv:1506.01509, 2015

[9] A. Choudhary, S. Rana, K. J. Matahai, "A Critical Analysis of Energy Efficient Virtual Machine Placement Techniques and its Optimization in a Cloud Computing Environment," International Conference on Information Security \& Privacy, 2015, Nagpur, INDIA Procedia Computer Science, 2016, pp. 132-138.

[10] V. Mann, A. Kumar, P. Dutta, S. Kalyanaraman, "VMFlow: Leveraging VM mobility to reduce network power costs in data centers", IBM technical report, NETWORKING 2011, 2011, pp. 198-211.

[11] K. Shi, H. Yu, F. Luo, G. Fan, "Multi-Objective Biogeography-Based Method to Optimize Virtual Machine Consolidation,” In SEKE 2016, pp. 225-230.

[12] S. Wang, Z. Liu, Z. Zheng, Q. Sun, F. Yang, "Particle swarm optimization for energy-aware virtual machine placement optimization in virtualized data centers," In Parallel and Distributed Systems, IEEE International Conference, 2013, pp. 102-109.

[13] D. Chang, G. Xu, L. Hu, K. Yang, "A network-aware virtual machine placement algorithm in mobile cloud computing environment," IEEE Wireless Communications and Networking Conference Workshops, 2013, pp. 117-122.

[14] D. Kakadia, N. Kopri, V. Varma, "Network-aware virtual machine consolidation for large data centers," In Proceedings of the $3^{\text {rd }}$ ACM International Workshop on Network-Aware Data Management, 2013, pp. 6.

[15] J. J. Prevost, K. Nagothu, B. Kelley, M. Jamshidi, "Optimal update frequency model for physical machine state change and virtual machine placement in the cloud," In System of Systems Engineering, $8^{\text {th }}$ IEEE International Conference, 2013, pp. 159-164.

[16] L. Shi, B. Butler, D. Botvich, B. Jennings, "Provisioning of requests for virtual machine sets with placement constraints in IaaS clouds," In Integrated Network Management, IFIP/IEEE International Symposium, 2013, pp. 499-505.

[17] K. Le, R. Bianchini, J. Zhang, Y. Jaluria, J. Meng, T. D. Nguyen, "Reducing electricity cost through virtual machine placement in high performance computing clouds," In Proceedings of 2011 ACM International Conference for High Performance Computing, Networking, Storage and Analysis, 2011, pp. 22.

[18] M. Sun, W. Gu, X. Zhang, H. Shi, W. Zhang, "A matrix transformation algorithm for virtual machine placement in cloud," In Trust, Security and Privacy in Computing and Communications, $12^{\text {th }}$ IEEE International Conference, 2013, pp. 1778-1783.

[19] M. Malekloo, N. Kara, "Multi-objective ACO Virtual Machine Placement in Cloud Computing Environments," IEEE workshop on Cloud Computing systems, Networks, and Applications, 2014, pp. 112-116.

[20] G. Wu, M. Tang, Y.C. Tian, W. Li, "Energy-Efficient virtual machine placement in data centers by genetic algorithm," $19^{\text {th }}$ international conference on Neural Information Processing, 2012, vol. 3, pp. 315-323.

[21] K. Li, H. Zheng, J. Wu, "Migration-based virtual machine placement in cloud systems," In Cloud Networking, $2^{\text {nd }}$ IEEE International Conference, 2013, pp. 83-90.

[22] J. T. Piao, J. Yan, "A network-aware virtual machine placement and migration approach in cloud computing," 
In Grid and Cooperative Computing, $9^{\text {th }}$ IEEE International Conference, 2010, pp. 87-92.

[23] C. C. Mark, D. Niyato, T. Chen-Khong, "Evolutionary optimal virtual machine placement and demand forecaster for cloud computing," In Advanced Information Networking and Applications, 2011 IEEE International Conference, 2011, pp. 348-355.

[24] Z. Zheng, R. Wang, H. Zhong, X. Zhang, “An approach for cloud resource scheduling based on Parallel Genetic Algorithm," In Computer Research and Development, $3^{\text {rd }}$ IEEE International Conference, 2011, vol. 2, pp. 444-447.

[25] J. Zhao, L. Hu, Y. Ding, G. Xu, M. Hu, "A heuristic placement selection of live virtual machine migration for energy-saving in cloud computing environment," PloS one. 2014 , vol. 9 , no. 9 .

[26] V. Kachitvichyanukul, "Comparison of three evolutionary algorithms: GA, PSO, and DE," Industrial Engineering and Management Systems, 2012, vol. 11, no. 3, pp. 215223.

[27] H. Kazemipoor, R. Tavakkoli-Moghaddam, P. Shahnazari-Shahrezaei, A. Azaron, "A differential evolution algorithm to solve multi-skilled project portfolio scheduling problems," The International Journal of Advanced Manufacturing Technology, 2013, pp. 10991111.

[28] V. Santucci, M. Baioletti, A. Milani, "Algebraic differential evolution algorithm for the permutation flowshop scheduling problem with total flowtime criterion," IEEE Transactions on Evolutionary Computation, 2016, vol. 20, no. 5, pp. 682-694.

[29] J. T. Tsai, J. C. Fang, J. H. Chou, "Optimized task scheduling and resource allocation on cloud computing environment using improved differential evolution algorithm," Computers \& Operations Research, 2013, vol. 40, no.12, pp. 3045-3055.

[30] D. Libao, W. Sha, J. Chengyu, H. Cong, "A Hybrid Mutation Scheme-Based Discrete Differential Evolution Algorithm for Multidimensional Knapsack Problem," In Instrumentation \& Measurement, Computer, Communication and Control, $6^{\text {th }}$ IEEE International Conference, 2016, pp. 1009-1014.

[31] A. Glotić, P. Kitak, J. Pihler, I. Tičar, "Parallel selfadaptive differential evolution algorithm for solving shortterm hydro scheduling problem," IEEE Transactions on Power Systems, 2014, vol. 29, no. 5, pp. 2347-2358.

[32] T. Liu, M. Maeda, "Set-Based Differential Evolution for Traveling Salesman Problem," In Intelligent Networks and Intelligent Systems, $6^{\text {th }}$ IEEE International Conference, 2013, pp. 107-110.

[33] K. Suresh, D. Kundu, S. Ghosh, S. Das, A. Abraham, S. Y Han," Multi-objective differential evolution for automatic clustering with application to micro-array data analysis," Sensors, 2009, vol. 9, no. 5, pp. 3981-4004.

[34] F. Lezama, A. Y. Rodríguez-González, E. M. de Cote, "Load pattern clustering using differential evolution with Pareto Tournament," In Evolutionary Computation, IEEE Congress, 2016, pp. 241-248.

[35] M. Zhao, R. Liu, W. Li, H. Liu, "Multi-objective optimization based differential evolution constrained optimization algorithm," In Intelligent Systems, $20102^{\text {nd }}$ IEEE WRI Global Congress, 2010 D, vol. 1, pp. 320-326.

[36] B. Rieck, "Basic Analysis of Bin-Packing Heuristics," Publicado por Interdisciplinary Center for Scientific Computing. Heildelberg University, 2010.

[37] J. Xu, J. A. Fortes, "Multi-objective virtual machine placement in virtualized data center environments," In Proceedings of the 2010 IEEE/ACM International
Conference on Green Computing and Communications \& International Conference on Cyber, Physical and Social Computing, 2010 Dec 18, pp. 179-188.

[38] R. Storn, K. Price, "Differential Evolution - A simple and efficient heuristic for global optimization over continuous spaces. Journal of global optimization, 1997, vol. 11, no. 4, pp. 341-59.

[39] J. Adeyemo, F. Bux, F. Otieno, "Differential evolution algorithm for crop planning: Single and multi-objective optimization model," International Journal of Physical Sciences, 2010, vol. 5, no. 10, pp. 1592-1599.

\section{Authors' Profiles}

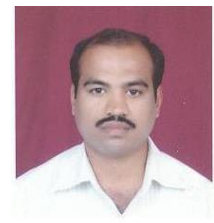

Dr. Amol C. Adamuthe received Ph.D. from Mumbai University and Master of Technology in Computer Engineering from Dr. B. A. Technological University, Lonere, MS, India. $\mathrm{He}$ is currently an Assistant Professor at Rajarambapu Institute of Technology, Sakharale, Sangli, MS, India. His areas of interest are technology forecasting, optimization and cloud computing.

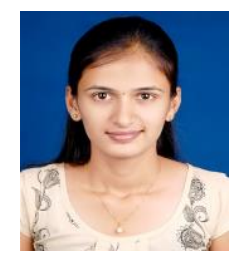

Miss. Jayshree T. Patil received the Bachelor of Engineering in Computer Engineering, from Mumbai University. She has completed M. Tech. in Computer Science and Engineering from Rajarambapu Institute of Technology, Sakharale, Sangli, MS, India. Her area of interest are cloud computing and optimization

How to cite this paper: Amol C. Adamuthe, Jayshree T. Patil, "Differential Evolution Algorithm for Optimizing Virtual Machine Placement Problem in Cloud Computing", International Journal of Intelligent Systems and Applications(IJISA), Vol.10, No.7, pp.58-65, 2018. DOI: 10.5815/ijisa.2018.07.06 